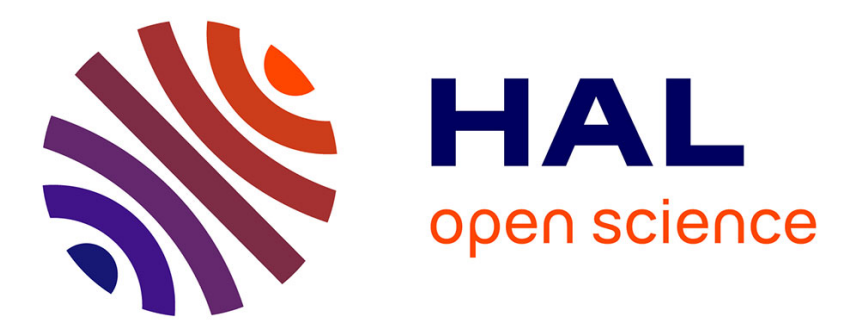

\title{
Réalisation d'un corrélateur électronique à lecture directe
}

\author{
J.C. Pebay-Peyroula, P. Simon
}

\section{To cite this version:}

J.C. Pebay-Peyroula, P. Simon. Réalisation d'un corrélateur électronique à lecture directe. J. Phys. Phys. Appl., 1954, 15 (S5), pp.101-108. 10.1051/jphysap:01954001505010100 . jpa-00212620

\section{HAL Id: jpa-00212620 https://hal.science/jpa-00212620}

Submitted on 1 Jan 1954

HAL is a multi-disciplinary open access archive for the deposit and dissemination of scientific research documents, whether they are published or not. The documents may come from teaching and research institutions in France or abroad, or from public or private research centers.
L'archive ouverte pluridisciplinaire HAL, est destinée au dépôt et à la diffusion de documents scientifiques de niveau recherche, publiés ou non, émanant des établissements d'enseignement et de recherche français ou étrangers, des laboratoires publics ou privés. 


\title{
RÉALISATION D'UN GORRÉLATEUR ÉLEGTRONIQUE A LEGTURE DIREGTE.
}

\author{
Par J. C. PEBAY-PEYROULA et P. SIMON, \\ Laboratoire de Physique de l'E. N.S.
}

\begin{abstract}
Sommaire. - On étudie un corrélateur qui donne à partir de deux enregistrements $F$ et $G$ la valeur de fonctions du type :

$$
R(\tau)=\frac{I}{\theta} \int_{0}^{0} F(t) G(t+\tau) \mathrm{d} t .
$$

L'appareil, grâce à un dispositif photoélectrique, utilise directement les bandes obtenues par des enregistreurs d'un type courant. La partie électronique calculatrice est du type à impulsions; elle ne limite pas la précision qui est imposée par la représentation graphique des enregistrements. Dans la réalisation actuelle, le temps de corrélation $\tau$ peut varier de façon continue à partir d'un minimum dépendant de la vitesse d'enregistrement jusqu'à des valeurs aussi grandes que l'on veut.
\end{abstract}

L'étude physique d'un phénomène conduit généralement à l'emploi des enregistreurs graphiques. L'enregistrement, dans le cas de phénomènes désordonnés, est la plupart du temps impropre à donner directement des résultats intéressants. Pour en déduire quelques propriétés, il est nécessaire de faire des calculs intermédiaires : développements de Fourier, recherche de fonction de corrélation. Dès que l'on doit dépouiller une certaine longueur d'enregistrement, ce travail devient long et fastidieux.

Aussi avons-nous imaginé un dispositif permettant de calculer de façon entièrement automatique à partir d'enregistrements des fonctions de la forme :

$$
f(\tau)=\frac{\mathrm{I}}{T} \int_{0}^{T} F(t) G(t+\tau) \mathrm{d} t,
$$

où $F$ et $G$ sont deux courbes tracées sur deux graphiques différents.

Nous pouvons ainsi obtenir rapidement les fonctions de corrélation de deux événements $F(t)$ et $G(t)$, les fonctions d'autocorrélation d'un événement $F(t)$ et si nous avons sur une bande une sinusoïde, des coefficients de Fourier.

Principe de l'appareil. - L'appareil comportera deux parties distinctes : la partie lectrice qui transformera les données graphiques en signaux électriques représentant dans un certain code les fonctions $F$ et $G$, et la partie électronique qui effectuera les calculs.

Partie lectrice. - La figure i nous montre un schéma général de l'appareil. On y voit les feuilles de papier d'enregistrement sur lesquelles sont tracées les fonctions $F$ et $G$ à étudier. Transversalement, le long du segment $A B$ se déplace sur le diagramme $F$ un point lumineux. Lorsqu'il arrive en B un autre apparaît en A et ainsi de suite. Une cellule photoélectrique est placée devant le papier. Lorsque la tache lumineuse passe sur le trait noir du graphique, la quantité de lumière diffusée varie et le courant de la cellule subit une impulsion. La vitesse de balayage étant uniforme et, par suite de la continuité du balayage, le courant cellule présente l'aspect de la figure 1.1. Un dispositif accessoire fournit à chaque balayage une impulsion définissant le zéro (fig. I.2). Entre une impulsion $a$ et une impulsion $b$, il s'écoule un temps proportionnel à $F(t)$.

A l'aide d'un second point lumineux et d'une deuxième cellule, nous faisons une lecture analogue en $\mathrm{A}^{\prime} \mathrm{B}^{\prime}$ sur le diagramme $G$. Nous obtiendrons des impulsions séparées par un temps proportionnel à $G(t+\tau)$.

Précisons dès maintenant que les vitesses de balayages sont différentes l'une de l'autre, nous expliquerons ce point ultérieurement.

Partie électronique. - Les impulsions $a$ et $b$ venant d'une même voie de lecture sont appliquées à un circuit flip-flop pour obtenir des ondes carrées. Le flip-flop ne peut prendre que deux états de fonctionnement que nous appellerons $\mathrm{A}_{1}$ et $\mathrm{B}_{1}$ (fig. I.3). L'impulsion $a$ lui est appliquée de façon à l'amener à la position $\mathrm{A}_{1}$ et à celle-ci seulement. De même, l'impulsion $b$ ne peut l'amener qu'à la position $\mathrm{B}_{1}$. La longueur de l'ordonnée sur le graphique est donc maintenant proportionnelle à la durée d'un état électrique, avec nos notations à la durée de l'état $\mathrm{A}_{1}$.

Nous pouvons appeler probabilité de l'événement $\mathrm{A}_{1}$ que nous écrirons prob $A_{1}$ le rapport de la durée de l'état $A_{1}$ à la durée totale 0 de l'opération. 
Faisons dérouler le papier à vitesse constante. Nous voyons sans peine que nous avons :

$$
\operatorname{prob} A_{1}=\frac{\boldsymbol{k}_{1}}{0} \int_{0}^{0} F(t) \mathrm{d} t,
$$

$K_{1}$ étant un facteur de proportionnalité.

Il en est de même de l'autre voie pour la lecture du diagramme $G$ et nous aurons en prenant des notations analogues :

$$
\text { prob t: }={ }_{1}^{k} \int_{11}^{0} G(t+\tau) \mathrm{d} t
$$

Supposons pour simplifier $F$ et $G$ constants et admettons que l'indépendance des deux balayages

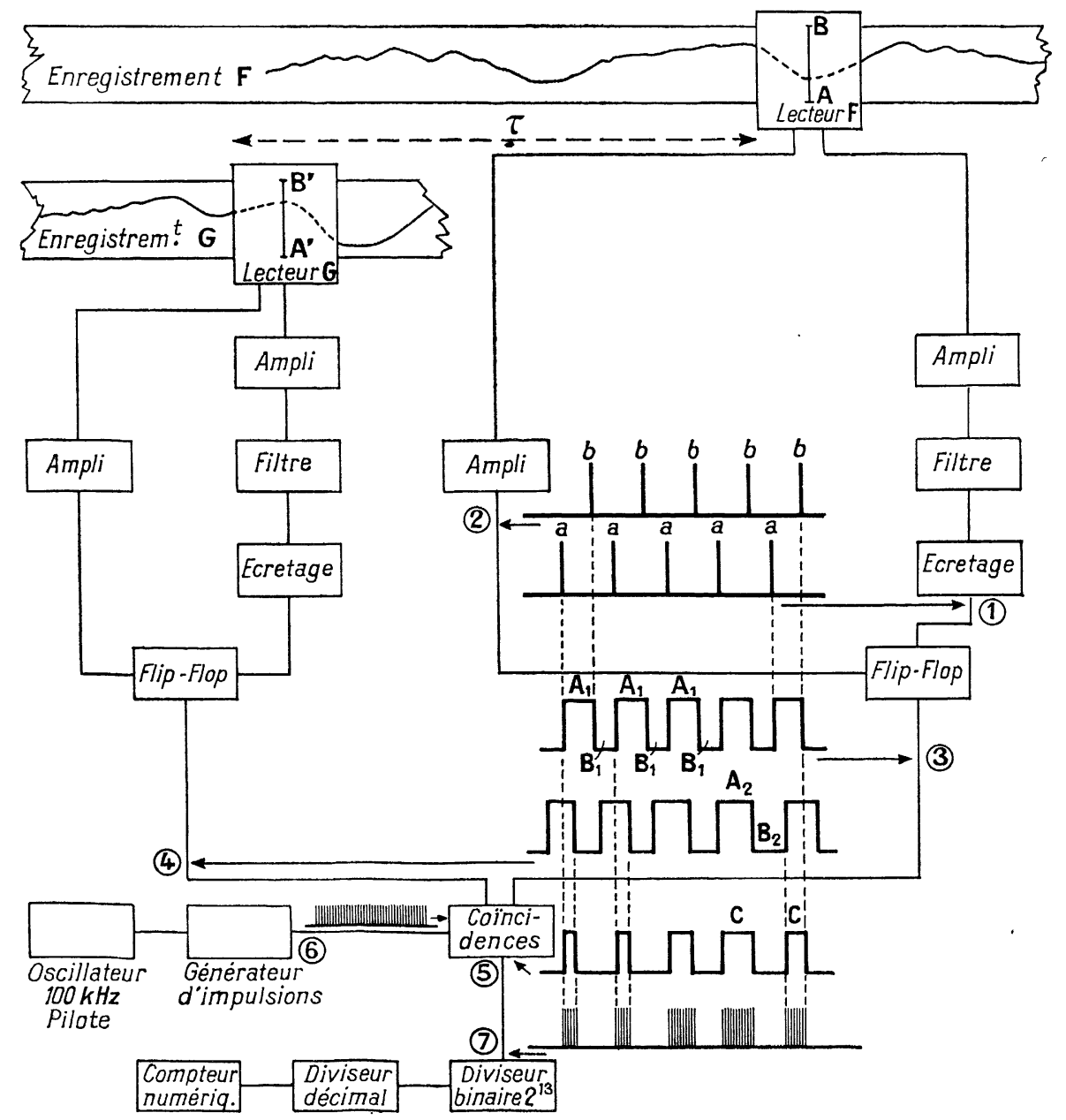

Fig. 1. - Schéma du corrélateur.

réalise entre les événements $A_{1}$ et $A_{2}$ une indépendance au sens du calcul des probabilités.

A l'aide d'un système électronique à coïncidence, fabriquons un état électrique $\mathrm{C}$ qui ne sera réalisé que lorsque les états $A_{1}$ et $A_{2}$ existeront simultanément (fig. I.5). Définissons pour $\mathrm{C}$ une probabilité du même type que celle définie pour $A_{1}$ et $A_{2}$. D'après le théorème des probabilités composées, nous aurons :

$$
\operatorname{prob} C=\operatorname{prob} A_{1} \operatorname{prob} A_{2} ;
$$

dans notre cas particulier avec $F$ et $G$ constants, nous avons :

$$
\begin{aligned}
& \operatorname{prob} A_{1}=K_{1} \boldsymbol{F}(t), \\
& \operatorname{prob} A_{2}=\boldsymbol{h}_{2} G(t+\tau),
\end{aligned}
$$

d'où

$$
\operatorname{prob} C=K F(t) G(t+\tau) \text {. }
$$

Nous admettons ici qu'en réalisant un balayage rapide tel que la variation des fonctions $F$ et $G$, considérées maintenant comme quelconques, soit très petite entre deux balayages successifs, nous 
aurons, l'opération étant menée du temps o au temps ()

$$
\operatorname{prob} C:=\frac{k}{0} \int_{11}^{0} r(1) G(1+\tau) d \%
$$

Pour mesurer prob $C$ un système à coïncidence ne laisse passer des impulsions venant d'un générateur d'impulsions que lorsque l'événement $\mathrm{C}$ est réalisé (fig. r.7). Ces impulsions sont comptées par un numérateur. Le rapport du nombre d'impulsions $n$ ainsi comptées au nombre total nous donne la valeur de prob $C$. Le générateur d'impulsions ayant une fréquence de répétition constante, on a

$$
n=h \frac{\mathrm{I}}{0} \int_{0}^{0} F(t) G(t+\tau) \mathrm{d} t .
$$

la constante $h$ pouvant être déterminée de façon expérimentale.

En répétant cette opération pour différentes valeurs de $\tau$ la courbe $n=f(\div)$ est la fonction de corrélation cherchée, au facteur multiplicatif $h$ près.

Une étude plus approfondie conduit à fixer les caractéristiques de l'appareil, compte tenu des possibilités techniques d'une part et de la précision cherchée d'autre part. Comme la lecture graphique impose une précision maximum de i $/ \mathrm{I}^{\mathrm{O}} \mathrm{o}$ de l'échelle, il suffit de donner à l'appareil des caractéristiques pour lesquelles la précision du calcul électronique sera toujours supérieure à $1 / 100^{\mathbf{e}}$, comme on l'a

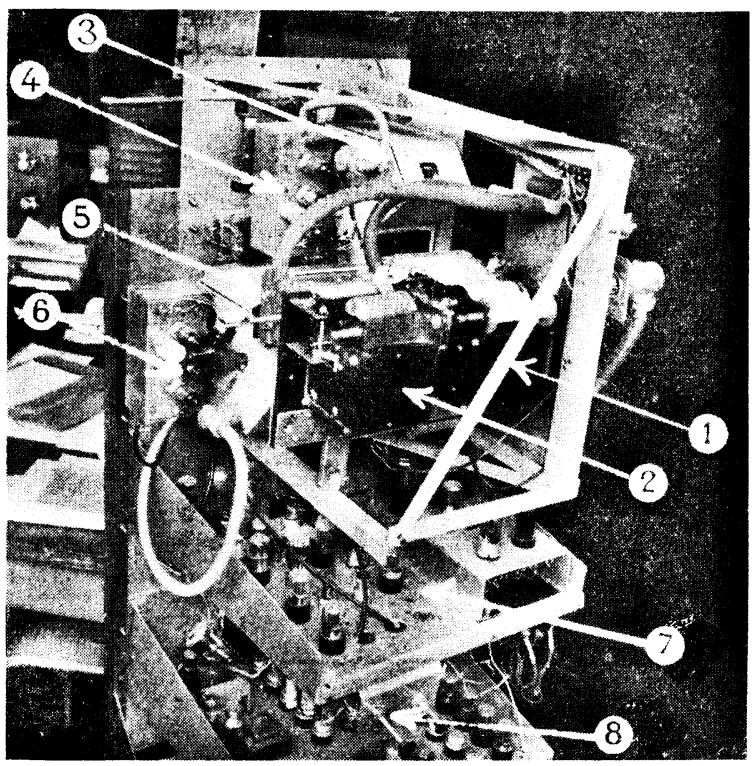

Fig. 2. - Réalisation.

1. Déroulement de l'enregistrement $F ; 2$. Déroulement de l'enregistrement $G$; 3 . Lecteur de F surmonté de son amplificateur 4 ; 5. Lecteur de G surmonté de son amplificateur 6; 7. Partie élect ronique calculatrice; 8. Diviseurs et compteur numérique. montré ailleurs (cf. Diplôme cité en bibliographie). On a été ainsi conduit aux valeurs suivantes :

Nombre de balayages par seconde : 300 à 500 ;

Vitesse de déroulement du papier : $/ 4 \mathrm{~mm} / \mathrm{s}$;

Fréquence de répétition du générateur d'impulsion : 100 ooo.

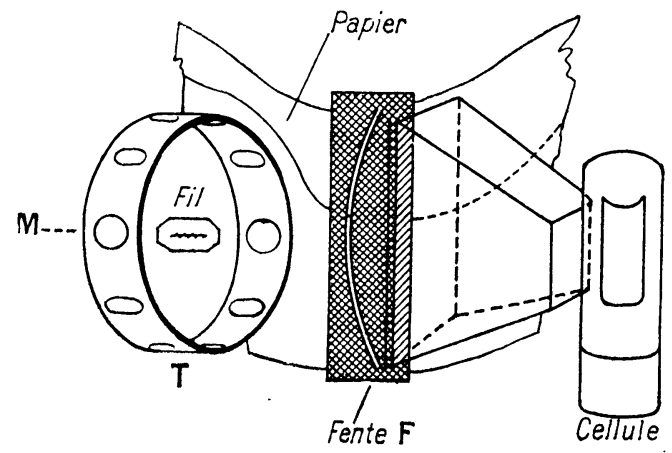

Fig. $3 a$. - Principe du lecteur.

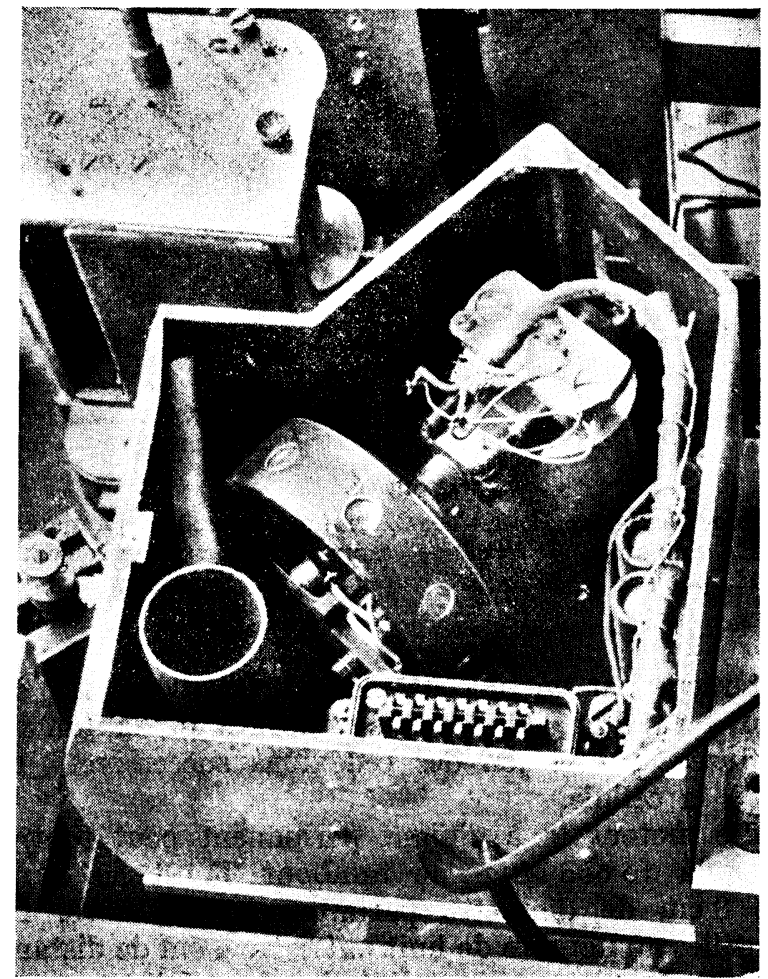

Fig. 3 b. - Partie mécanique du lecteur.

Réalisation (fig. 2). - PARTIE Lectrice. - Le corrélateur a été réalisé pour les besoins d'un laboratoire déterminé. La plupart des enregistrements y sont faits sur l'enregistreur Pekly, nous avons dû adapter la partie lectrice aux caractéristiques de cet appareil. L'axe de la bande de papier est l'axe des 
temps. L'axe de la fonction est une partie d'arc de cercle.

Il faudra donc que la tache lumineuse décrive d'un mouvement uniforme une portion d'arc de cercle en satisfaisant aux conditions de continuité. La réalisation mécanique devra, d'autre part, être telle que l'on puisse rapprocher le plus près possible les deux lecteurs si l'on veut faire un travail d'autocorrélation pour des temps ? très courts.

Après de nombreux tâtonnements, nous avons réalisé les lecteurs de courbe de la façon suivante (fig. $3 a$ et $b$ ).

Un moteur $\mathrm{M}$ entraîne un tambour $\mathrm{T}$ à la périphérie duquel est placé un certain nombre de lentilles. $\mathrm{Au}$ centre du tambour est placée une ampoule électrique rectiligne. L'axe du filament est placé suivant l'axe du tambour. L'image du filament se forme sur une fente $\mathrm{F}$, les bords sont recouverts de noir optique. Derrière la fente se déplace le papier d'enregistrement. La lumière diffusée par le papier apparaissant derrière la fente

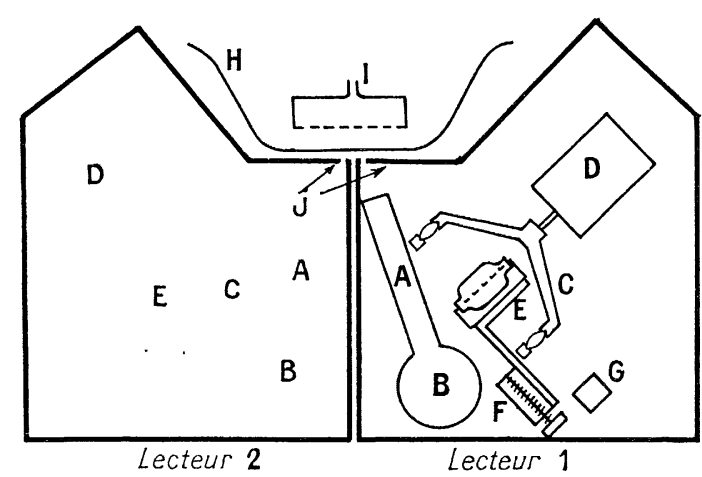

Fig. 4.

A, bloc plexiglass; B, cellule ; C, tambour à lentilles; D, moteur; $\mathrm{E}$, ampoule; $\mathrm{F}$, réglage ampoule; $\mathrm{G}$, phototransistor; $\mathrm{H}$, papier; I, dispositif pneumatique; J, fentes.

est reçue par un bloc de plexiglass taillé en trapèze qui " conduit " la presque totalité de la lumière diffusée, par le jeu des réflexions successives, jusqu'à la cellule.

Le moteur $\mathrm{M}$ à aimant permanent peut tourner jusqu'à ıo ooo $\mathrm{t} / \mathrm{mn}$, le tambour $\mathrm{T}$ est en dural, de $8 \mathrm{~cm}$ de diamètre parfaitement équilibré. Les lentilles au nombre de huit ont donc $2 \mathrm{~cm}$ de distance focale. La presque totalité du flux diffusé par le papier étant reçue par la cellule, grâce au bloc de plexiglass, nous avons pu nous servir d'une cellule ordinaire à vide. En tenant compte du fait que nous devons avoir deux lecteurs pouvant effectuer des lectures très rapprochées, nous arrivons à la réalisation définitive schématisée par la figure 4 . De cette façon, pour un enregistrement fait à la vitesse de $5 \mathrm{~mm} / \mathrm{s}$, l'écart minimum de $2 \mathrm{~mm}$ correspond à $:=2 / 5 \mathrm{e}$ s.
Le dispositif accessoire fournissant une impulsion de zéro dont nous avons parlé dans la première partie est constitué par un phototransistor. Ce dernier est placé de telle façon que l'image du filament se forme sur la partie sensible à l'instant désiré. En changeant la position du phototransistor,

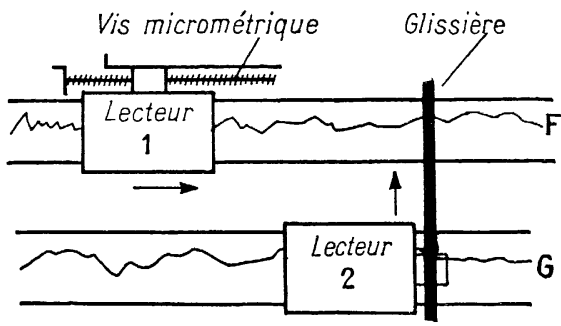

Fig. 5. - Déplacement des lecteurs, le sens de déplacement est marqué par des flèches.

on fixe le zéro au niveau correspondant à l'enregistrement étudié. Nous avons choisi pour cet emploi un phototransistor au germanium car sa partie sensible très réduite $\left(0,5 \mathrm{~mm}^{2}\right)$ permet d'obtenir très simplement des impulsions et son peu d'encombrement simplifie la réalisation.

Les diagrammes se déroulent en synchronisme à la vitesse constante de $4 \mathrm{~mm} / \mathrm{s}$ obtenue à l'aide d'un moteur synchrone. Le papier est parfaitement appliqué contre les bords de la fente à l'aide d'un système pneumatique.

La figure 5 schématise les déplacements des lecteurs permettant d'une part de faire varier - et d'autre part de faire soit de la corrélation, soit de l'autocorrélation.

Partie Électronique. - Elle est schématisée par la figure i. Les réalisations étant classiques, nous' ne donnerons que les précisions qui nous semblent nécessaires.

Le courant donné par l'amplificateur de cellule

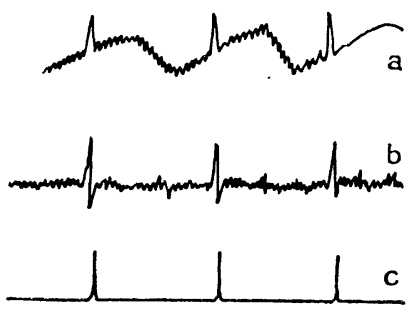

Fig. 6.

placé directement sur le lecteur présente une modulation parasite de fréquence égale à celle du balayage (fig. $6 a$ ). Un filtre passe-haut élimine cette modulation (fig. $6 \mathrm{~b}$ ), un écretage permet de ne conserver que les impulsions et élimine un certain nombre 
d'impulsions parasites de niveau moins élevé (parasites de moteurs, etc.) (fig. 6c).

Les coïncidences successives sont réalisées dans un seul circuit représenté par la figure 7 . Jes diodes

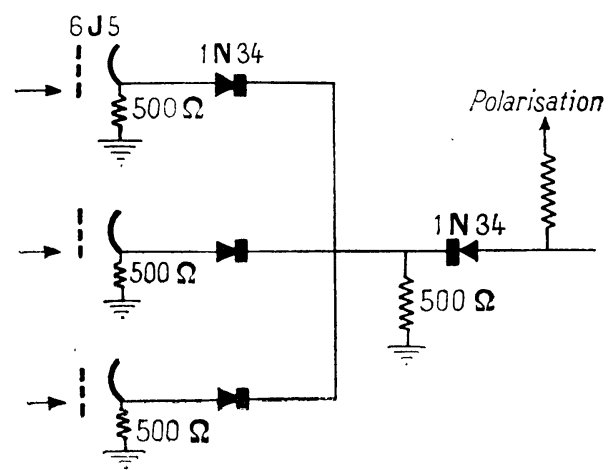

Fig. 7 .

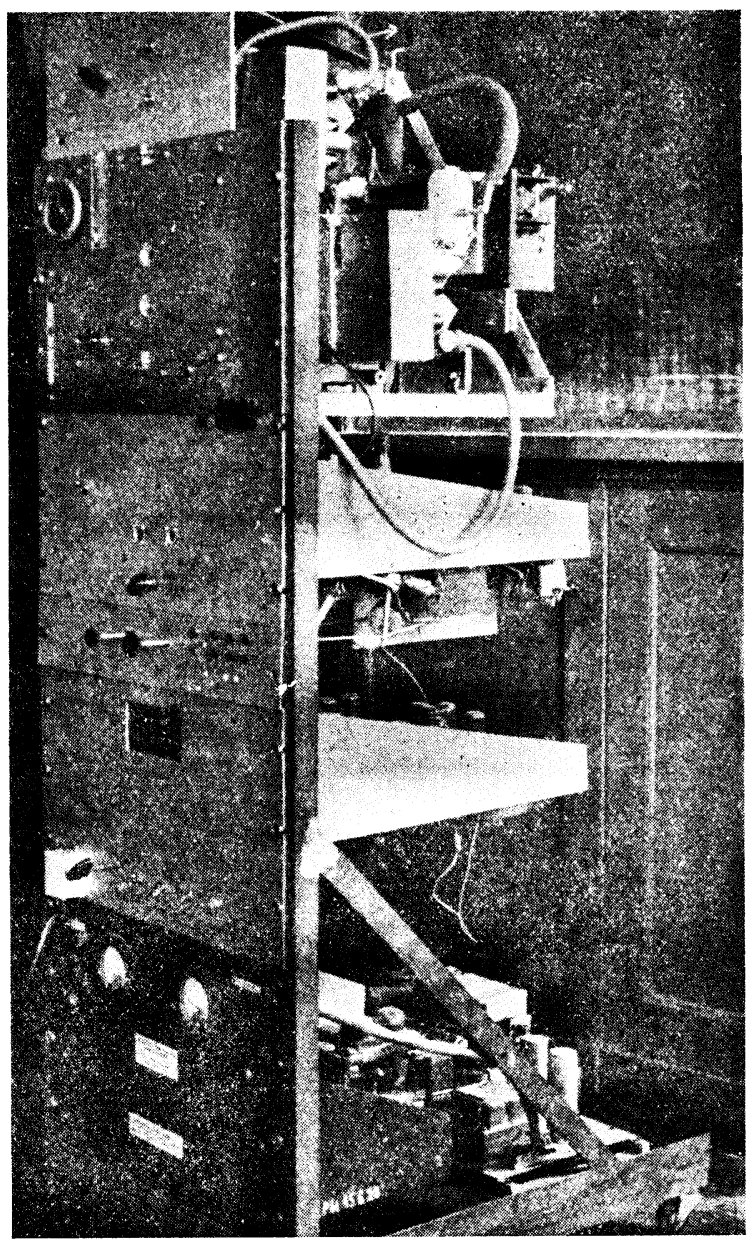

Fig. 8. - Réalisation d'ensemble.

sont disposées de telle façon que seules les impulsions correspondant à la coïncidence des états $A_{1}$ et $\mathrm{A}_{2}$ puissent passer. Les numérateurs sont réalisés de façon classique.

Des découplages soignés de haute tension ont été nécessaires pour éviter toute réaction des différents circuits les uns sur les autres. La figure 8 nous montre la réalisation d'ensemble provisoire.

La mise au point définitive s'est avérée très délicate

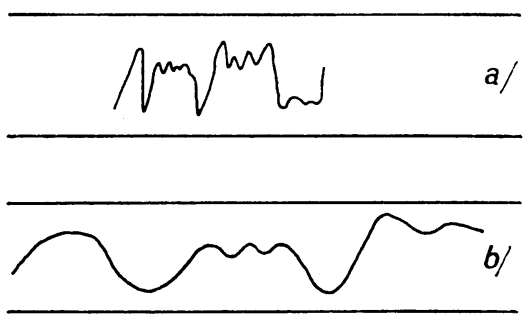

Fig. 9. - a. Courbe inutilisable; $b$. la même "étalée " utilisable.

dans de nombreux points de détail. Signalons, par exemple, que les courbes doivent être tracées sur un papier ne présentant aucune inscription ni ligne. La courbe doit être tracée à l'encre noire et avoir un trait d'un demi-millimètre de large. L'enregistrement du phénomène étudié doit être assez rapide pour que la courbe ne présente pas de variation trop brutale d'amplitude (fig. 9). Moyennant ces quelques précautions, remarquons que ce corrélateur travaille directement sur les courbes enregistrées pour fournir immédiatement des résultats chiffrés pour chaque valeur de $\tau$ étudiée.

Des vérifications ont été faites sur des courbes dont on peut déterminer rapidement par le calcul

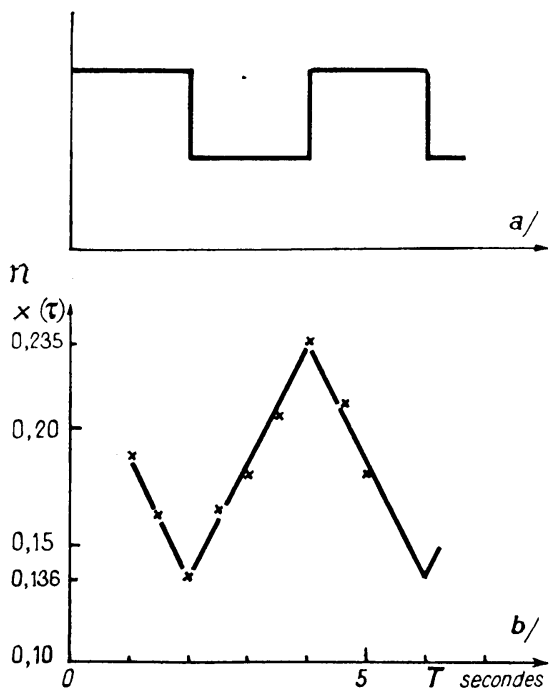

Fig. 10.

a. Fonction $F$;

$b$. Fonction $n(\tau)$ et points obtenus avec le corrélateur.

$n(\tau)=\frac{1}{t} \int_{0}^{t} F(t) F(t+\tau) \mathrm{d} t$

[En ordonnées, lire $n(\tau)]$. 
la fonction de corrélation. La figure to nous donne par exemple une de ces courbes, la fonction d'autocorrélation calculée et les points obtenus de façon expérimentale. On peut donc attendre une bonne précision de cet appareil.

Ce travail a été réalisé à la demande du laboratoire de Radioastronomie de l'École Normale Supérieure qui nous a apporté, sans aucune restriction, toute l'aide matérielle nécessaire.

Nous tenons à remercier M. le Professeur Rocard, directeur du Laboratoire de Physique qui a permis la réalisation de ce travail, ainsi que $M$. Aigrain qui nous a dirigé pour la construction de cet appareil.

\section{APPENDIGE.}

Méthode utilisée pour déterminer les paramètres en fonction de la précision cherchée. Nous avons supposé dans l'exposé précédent que les événements $A$ et $B$ étaient indépendants au sens du calcul des probabilités. Les événements A et B sont fonction de la vitesse de rotation des deux moteurs. II nous faut justifier l'application du théorème des probabilités.

La méthode d'étude qui nous a paru la plus simple est une méthode directe dont nous allons montrer les étapes. Rappelons avant de commencer qu'une précision de calcul supérieure à $\mathrm{I} / \mathrm{IOO}^{\mathrm{e}}$ est illusoire, les fonctions n'étant tracées graphiquement qu'à ce degré de précision près.

Diagramme des aires. - Pendant un temps élémentaire $\Delta t$ l'événement $\mathrm{C}$ a une certaine probabilité $y$. Nous portons $y$ en ordonnée et le temps

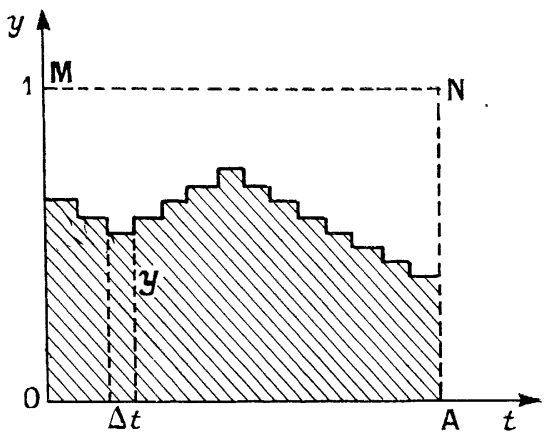

Fig. I I.

en abscisse. De o à $t$ la probabilité moyenne $\stackrel{o-\overline{\text { prob }}^{-T} \text { est }}{\text { est }}$ évidemment égale au rapport de l'aire hachurée à celle du rectangle OAMN (fig. i I).

Évaluation directe de prob $C$ dans un cas simple. - Nous allons montrer comment nous pouvons résoudre le problème dans un cas simple : les deux moteurs tournent à vitesse constante, ces vitesses sont différentes mais dans un rapport de la forme $\frac{n}{n+\mathrm{I}} n$ étant un entier simple et les fonctions $F$ et $G$ ont des valeurs constantes $f$ et $g$. Sur la figure nous avons représenté les événements A et $\mathrm{B}$ correspondants, nous voyons apparaître une " période " $T$. Nous pouvons, de façon analytique, en fonction des différentes données, évaluer prob $C$ pour des temps élémentaires $\Delta t$ et faire un diagramme des aires que nous avons dessiné (fig. I2) avec la

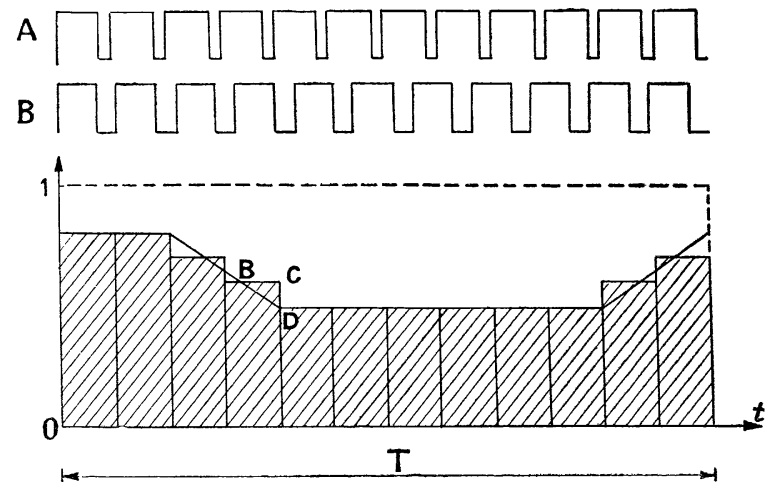

Fig. 12.

même échelle des temps. A partir des données analytiques définissant les points nous pouvons évaluer la valeur moyenne de probC pour une période. Si $\Delta t$ est petit et permet de confondre l'aire de la figure avec l'aire du polygone, nous obtenons ce résultat très intéressant

$$
o \overline{\operatorname{prob} C}{ }^{r}=f g \text {; }
$$

le diagramme des aires peut d'ailleurs prendre des formes diverses pour des valeurs variées de $f$ et $g$.

Étude de la précision dans ce cas simple : première approximation (fig. ı3). - Supposons que nous voulions calculer l'intégrale

$$
\frac{\mathrm{I}}{t} \int_{0}^{t} f g \mathrm{~d} t
$$

qui se ramène comme $f$ et $g$ sont constants au produit $f g$. Le diagramme des aires nous donne une méthode d'évaluation. Si l'unité de temps est la durée d'une période, nous avons

$$
f_{\mathcal{S}}=\frac{\text { aire OAMN }}{t} \text {. }
$$

Cette quantité est en définitive une fonction de $t$ (fig. I $3 a$ ).

En faisant varier $t$, la fonction

$$
\text { aire OAMN }=f(t)
$$

est représentée par la figure ı $3 b$. 
La figure i $3 c$ nous montre la variation de la fonction $f g$ elle-même en fonction de l'instant final. Pour être sûr d'avoir une précision du $\mathbf{I} / \mathrm{I}_{00} \mathbf{e}^{\mathrm{e}}$

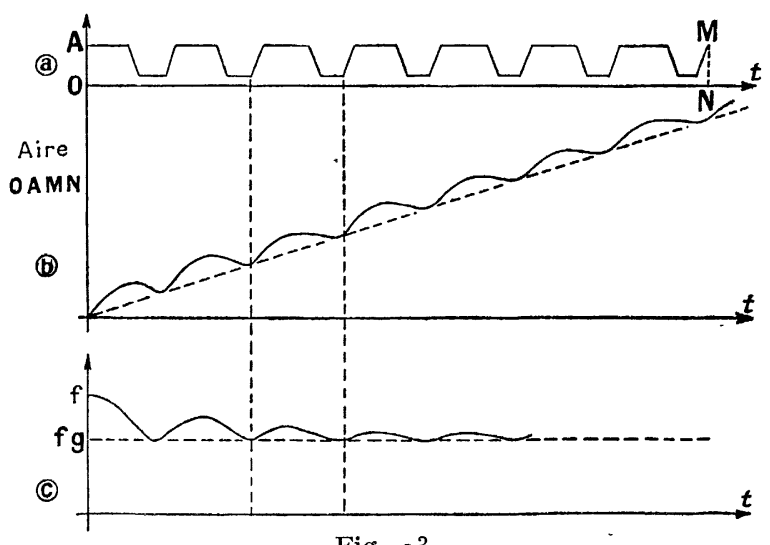

Fig. I 3.

quelque soit l'instant final, il faut que l'amplitude des variations soit inférieure à $\mathrm{I} / \mathbf{1} \mathrm{OO}^{\mathrm{\theta}}$. Nous avons calculé la précision obtenue, en valeur absolue pour un certain nombre de valeurs de $f$ et $g$ ( $f i g$. 14).

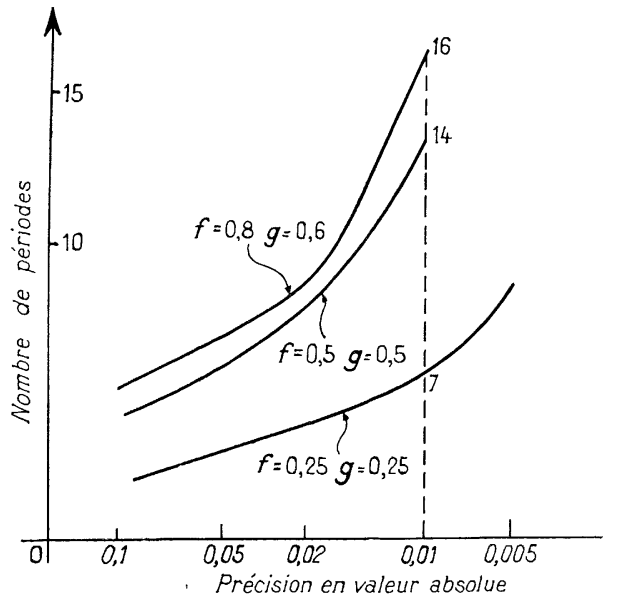

Fig. 14.

Galcul de la précision dans le même cas : deuxième approximation. - Nous avons évalué précédemment l'aire correspondant à une période sans tenir compte des valeurs finies du $\Delta t$. L'erreur commise est au maximum égale à l'aịre de deux triangles tels que BCD (fig. 1 2). Cette erreur est de l'ordre de $\frac{\mathrm{I}}{n^{2}}, n$ étant le nombre de balayages par période. Nous pouvons affirmer dans notre cas particulier qu'en prenant 20 périodes comprenant chacune ro balayages, la précision obtenue est suffisante dans tous les cas.

Généralisation de la méthode. $-\mathrm{I}^{\circ}$ Une période telle que celle vue précédemment suppose un rapport entier simple entre les deux vitesses de moteurs. En réalité il n'en est jamais ainsi, mais un exemple va nous montrer que l'on peut traiter de façon analogue le problème quelque soit le rapport des vitesses de moteurs. La figure 15 a montre le

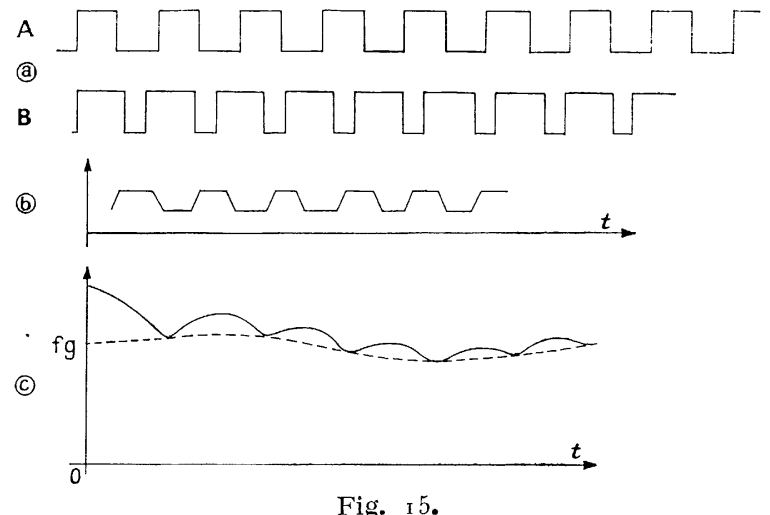

cas de vitesses respectives de $2500 \mathrm{t} / \mathrm{mn}$ (1) et $2940 \mathrm{t} / \mathrm{mn}$ (2). Tous les balayages du moteur (1) le diagramme des aires prend l'aspect d'une " période » précédente, mais toutes les périodes n'ont pas exactement la même aire. Un calcul montre que l'on peut négliger, au bout de quelques périodes, ce phénomène ( $f i g$. I $5 \mathrm{c}$ ).

Nous avons besoin au maximum d'une précision de $\mathbf{1} / \mathbf{I}^{\circ}{ }^{\mathbf{e}}$. Il est donc inutile d'avoir des périodes ayant plus de ı balayages : on augmenterait inutilement la longueur des opérations.

${ }_{2}$ o Supposons maintenant que, les fonctions $F$ et $G$ varient. Pour plus de simplicité, supposons une variation linéaire de $F$ pendant une période $T$. On trace le diagramme des aires dans ce cas (fig. I6)

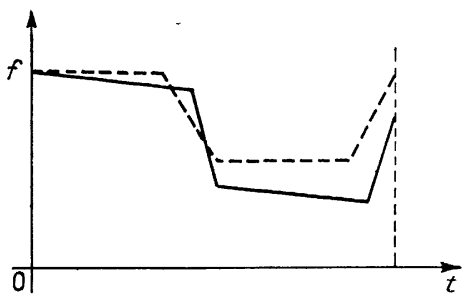

Fig. 16.

pour calculer directement probC. L'évaluation de l'aire est assez difficile et conduit à des termes du deuxième ordre de la forme $(\Delta f)^{2}$. On montre que l'on peut les négliger si la variation $\Delta f$ est inférieure à $1 / 1 o^{\mathbf{e}}$. Dans ce cas on trouve la valeur simple

$$
o \overline{\operatorname{prob} C}^{T}=\frac{\mathrm{I}}{T} \int_{0}^{T} f g \mathrm{~d} t .
$$

Le travail de corrélation étant commencé depuis 
un certain temps, l'importance des fluctuations dues à chaque période est très faible et si l'on fait varier $F$ et $G$ de moins de $\mathrm{I} / \mathrm{I}^{\mathrm{e}}$ par période (les fonctions $F$ et $G$ sont normées par rapport à I) nous aurons finalement

$$
\frac{\mathrm{I}}{\theta} \int_{0}^{\theta} f(t) g(t+\tau) \mathrm{d} t \quad \grave{a ̀ ~} \mathbf{I} / \mathbf{I O O}^{\mathrm{e}} \text { près. }
$$

On peut étudier d'une façon analogue l'influence des fluctuations des vitesses des moteurs.

Rapidité du calcul. - Nous allons appliquer les considérations précédentes au calcul de la vitesse possible du déroulement de papier dans un exemple précis. Une fonction subit un accroissement correspondant à $\mathrm{I} \mathrm{cm}$ d'enregistrement et une variation

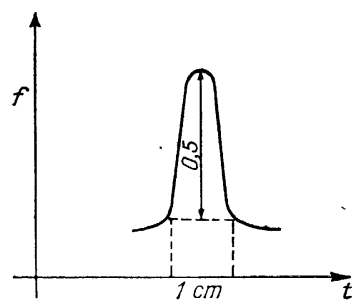

Fig. 17 . de niveau de o,כ ( $f i g .17$ ). Une période devra durer pendant au maximum une variation de niveau de $o, \mathrm{I}$, soit pendant I $\mathrm{mm}$ d'enregistrement. Si l'on règle les vitesses des moteurs pour avoir 200 balayages/s, une période peut durer $\mathrm{I} / 2 \mathrm{O}^{\mathrm{e}} \mathrm{S}$. On peut donc opérer avec une vitesse de $20 \mathrm{~mm} / \mathrm{s}$, soit environ $1 \mathrm{~m} / \mathrm{mn}$.

Il est à remarquer que nous avons estimé des limites supérieures d'erreur prises en valeur absolue. Le signe des erreurs commises localement, c'està-dire pour une brève longueur de l'enregistrement ne peut être déterminé a priori et sur une grande longueur d'enregistrement, l'erreur finale sera beaucoup plus petite par suite de la compensation des signes. Nous avons donc évalué une limite supérieure de l'erreur.

En définitive, seul le tracé de l'enregistrement graphique limite la précision. Un enregistrement de plus grande taille, donc plus précis, serait difficile à utiliser par le même procédé. On pourrait peutêtre envisager dans ce cas un dispositif de transcription qui, piloté à la main donnerait une fois pour toutes sur un ruban magnétique une traduction codée comme celle fournie par les lecteurs. Deux lecteurs magnétiques joueraient ensuite le rôle des lecteurs à cellule de l'appareil actuel.

Manuscrit reçu le i 5 octobre 1953 .

BIBLIOGRAPHIE

\section{Fonctions aléatoires \\ et fonctions de corrélation en général.}

James, Nichols N. B. et Philipps R. S. - Theory of Servomecanisme. Mass. Inst. Techn. Rad. Lab. Séries.

Rice. - A Mathematical analysis of Random Noise. - Bell Tech. J., г $944, \mathrm{n}^{\circ} 23$; $1945, \mathrm{n}^{\circ} 24$.

\section{Emplois des fonctions de Corrélations en Radioastronomie.}

Blum E. J. et Denisse J. F. - C. R. Acad. Sc.,ig5o, 231, г 2 I 4 .

Blum E. J., Denisse J. F. et Steinberg J. L. - $C . R$ Acad. Sc., $195 \mathrm{I}, \mathbf{2 3 2}, 388$.
Réalisation d'un corrélateur.

Singleton H. E. - A digital electronic correlator. Proc. Inst. Radio Eng., I95o, p. 1422 .

Brooks F. E. et Smith H. W. - A Computer for correlation functions. Rev. Sc. Instr., I 952, p. $12 \mathrm{I}$.

Pebay-Peyroula J. C. - Étude et réalisation d'un corrélateur électronique. Diplôme d'Études sup., 1952.

\section{Ouvrages techniques.}

Change B., Hughes V., Mc Nichol E., SAyre D. et Williams F. C. - Wave Forms. Mass. Inst. Techn. Rad. Lab. Séries. Méthode de multiplication de Hardy, p. 676; Échelles et numérateurs, p. 6o4.

Naslin et Peuteman. - Les compteurs électroniques d’impulsions. Onde électrique, 1949, nos 267 et 269. 